\title{
Up-regulated claudin 7 expression in intestinal-type gastric carcinoma
}

\author{
JEONG YOUP PARK $^{1}$, KYUNG HWA PARK ${ }^{1}$, TAE YUN OH ${ }^{1}$, SUNG PIL HONG ${ }^{1}$, TAE JOO JEON ${ }^{1}$, \\ CHANG HOON KIM ${ }^{1}$, SEUNG WOO PARK ${ }^{1}$, JAE BOCK CHUNG ${ }^{1}$, SI YOUNG SONG ${ }^{1,2}$ and SEUNGMIN BANG ${ }^{1}$ \\ ${ }^{1}$ Division of Gastroenterology, Department of Internal Medicine, Yonsei Institute of Gastroenterology, \\ Yonsei University College of Medicine, Seoul; ${ }^{2}$ Brain Korea 21 Project for Medical Science, Korea
}

Received February 20, 2007; Accepted April 23, 2007

\begin{abstract}
The disruption of tight junction protein expression in cancer might account for invasiveness, loss of cohesion, and lack of differentiation. Our cDNA array data indicated that claudin 7 was up-regulated in gastric carcinoma. We investigated the expression patterns and clinical implications of claudin 7 in gastric cancer. By immunohistochemical staining and Western blot, claudin 7 was significantly more often expressed in intestinal metaplasia, adenoma and cancer than in normal gastric epithelium. Twenty-seven (47.4\%) of 57 normal gastric epithelium samples did not express claudin 7 , but $50(86.2 \%)$ of 58 intestinal metaplasia, $11(91.7 \%)$ of 12 adenoma tissues, and $129(82.7 \%)$ of 156 cancer samples did. Claudin 7 was more often unexpressed in diffuse type gastric cancer than in intestinal type. Only $13(11.2 \%)$ of 116 intestinal type samples did not express claudin 7, but 14 (41.2\%) of 34 diffuse type samples showed no expression. Compared to normal gastric epithelium, intestinal type gastric cancer significantly more often expressed claudin 7, but diffuse type did not. The expression pattern of claudin 7 did not change as cancer progressed. In this study we show that claudin 7 expression changed with the gastric carcinogenic process and that this is implicated in cancer characteristics.
\end{abstract}

\section{Introduction}

Tight junctions encircle cells at the apical end of epithelial and endothelial lateral membranes. They are important for the barrier properties of these tissues and also contribute to cell surface polarity $(1,2)$. Recent studies have found that they recruit signaling molecules involved in cell proliferation, differentiation and gene expression. It has been suggested that

Correspondence to: Dr Seungmin Bang, Division of Gastroenterology, Department of Internal Medicine, Yonsei University College of Medicine, 134 Shinchon-Dong, Seodaemun-Ku, 120-752 Seoul, Korea

E-mail: bang7028@yumc.yonsei.ac.kr

Key words: tight junction protein, claudin 7, gastric carcinoma and microarray the disruption of tight junction protein in cancer might account for invasiveness, loss of cohesion and lack of differentiation $(3,4)$.

Claudins are transmembrane proteins with extracellular loops that are potential targets for antibody-based diagnostic and therapeutic modalities (4). Given that tumorigenesis is associated with the disruption of tight junctions, downregulation of claudins is expected. However, studies of claudins in malignancy are limited and rather confusing. Claudins 1 and 7 are down-regulated in breast, colon, and head and neck cancer (5-7), but other claudin proteins are actually upregulated in ovarian, breast, prostate and pancreatic cancer (8-10). Some studies have been able to demonstrate a correlation among claudin proteins and tumor cell differentiation, progression and prognosis $(5,11)$. However, some claudins have cancer-specific functions. For example, expression of claudin 4 in pancreatic cancer reduces invasiveness (12), but the same claudin in intraductal papillary mucinous neoplasm is associated with a more invasive phenotype (13).

Recently, using cDNA array experiments, we identified claudin 7 as being highly up-regulated in gastric adenoma and carcinoma. Like other claudin proteins, the function of claudin 7 is not well known. In mouse models, claudin 7 is expressed throughout the duodenum, jejunum and colon (14). Reduced claudin 7 expression in breast cancer correlates with higher tumor grade and metastatic disease (15). Claudin 7 expression is reportedly an early event in gastric carcinogenesis (16). Here, we investigated the protein expression patterns and clinical implications of claudin 7 in gastric carcinoma.

\section{Materials and methods}

Microarray analysis. Ten normal gastric tissue samples, 7 intestinal-type gastric cancer samples, 2 diffuse-type gastric cancer samples, and 5 mixed-type gastric cancer samples were frozen in liquid nitrogen and homogenized with a polytron (Kinematica Inc., Newark, NJ, USA). Total-RNA from normal tissues and cancer tissues was isolated using TRIzol reagent (Gibco-BRL, Grand Island, NY, USA) and purified using the RNeasy mini kit and the RNase free DNase set (Qiagen, Valencia, CA, USA) according to the manufacturer's protocols. RNA quality of all samples was tested by RNA electrophoresis and RNA LabChip analysis (Agilent, Palo Alto, CA, USA) to ensure RNA integrity. 
cDNA from each sample was synthesized with the Superscript cDNA synthesis kit (Invitrogen, Carlsbad, CA, USA) using the T\&-(dT)24 primer instead of the oligo(dT) provided in the kit. Biotin-labeled cRNA was then transcribed in vitro from cDNA using the BioArray HighYield RNA transcript labeling kit (Enzo Biochem, New York, NY, USA) and purified with the RNeasy Mini Kit. Purified cRNA was fragmented by incubation in fragmentation buffer [ $40 \mathrm{mmol} / \mathrm{l}$ trisacetate (pH 8.1), $100 \mathrm{mmol} / \mathrm{l} \mathrm{KOAc}, 30 \mathrm{mmol} / \mathrm{l} \mathrm{MgOAc}]$ at $95^{\circ} \mathrm{C}$ for $35 \mathrm{~min}$ and chilled on ice. Fragmented, labeled cRNA was tested on a Test Chip (Affymetrix, Santa Clara, CA, USA) to ensure that the control transcript $3^{\prime} / 5^{\prime}$ ratio was $\sim 1$. Fragmented, labeled cRNA was then applied to the Human Genome U133A Array (Affymetrix) and hybridized to the probes in the array. After washing and staining, the arrays were scanned. Two independent experiments were conducted to verify the reproducibility of results.

Gene expression levels were normalized and analyzed using the Microarray Suite, MicroDB, and Data Mining Tool software (Affymetrix). The average differential expression of 22215 genes, the absolute call difference, fold change and average differential expression between two or several samples were normalized and determined using this software package. Clustering and annotation of gene expression were analyzed using Cluster and Tree View (17) and GenMapp (18). Genes that were not annotated or easily classified were excluded from the clustering analysis.

To verify alterations in gene expression at the mRNA level, select genes with varying expression profiles were assayed by real-time reverse transcription-PCR analysis. Two micrograms of total-RNA from each sample were subjected to reverse transcription using the Superscript firststrand cDNA synthesis kit (Invitrogen) according to the manufacturer's protocol. Real-time PCR was then carried out in a total of $25 \mu 1$ (2 $\mu 1$ of cDNA, $12.5 \mu 1$ of $2 X$ SYBR Green PCR Master Mix, $1.5 \mu \mathrm{l}$ of each $5 \mu \mathrm{mol} / \mathrm{l}$ forward and reverse primers, and $7.5 \mu \mathrm{l}$ of $\mathrm{H}_{2} \mathrm{O}$ ) in SmartCycler II (Cepheid, Sunnyvale, CA, USA). The PCR program was initiated by $10 \mathrm{~min}$ at $95^{\circ} \mathrm{C}$ before 40 thermal cycles of $15 \mathrm{sec}$ at $95^{\circ} \mathrm{C}$ and $1 \mathrm{~min}$ at $60^{\circ} \mathrm{C}$. PCR amplification efficiency and linearity for each gene, including targeted and control genes, were tested. Data were analyzed according to the comparative $\mathrm{C}_{\mathrm{t}}$ method and normalized against $B$-actin or glyceraldehyde-3-phosphate dehydrogenase expression in each sample.

Immunohistochemical staining. A set of gastric cancer and adenoma samples were randomly obtained from individuals who had undergone gastrectomy for gastric cancer or endoscopy for adenoma between January 1999 and Decemder 2001 at Severance Hospital, Yonsei University College of Medicine. All patients underwent gastrectomy for curative purposes and gave informed consent for using tissue samples. One hundred and sixty-eight cancer and 13 adenoma tissue samples were collected. Sixty-two normal gastric epithelium samples and 58 intestinal metaplasia samples obtained from tissues adjacent to tumors were also collected. A pathological grading was made, and the tumor stage of the tissue samples was determined according to the AJCC staging system. After pathological evaluation, a tissue microarray (TMA) was generated using cores from tumors and adjacent normal tissue from each specimen. The Ethics Committee for the Clinical Research of the Institutional Review Board of Yonsei Medical Center, Korea, approved this study protocol.

Immunostaining with a goat-derived polyclonal claudin 7 antibody (Santa Cruz Biotechnology, Santa Cruz, CA, USA) at a dilution of 1:100 was performed on TMAs, using standard procedures. Initially, TMA slides were deparaffinized in xylene and rehydrated in graded alcohol. Endogenous peroxidase activity was blocked by incubating slides with methanol containing $0.3 \%$ hydrogen peroxide at room temperature for $20 \mathrm{~min}$. Microwave antigen retrieval was performed in citrate buffer (0.01 M, pH 6.0) for $10 \mathrm{~min}$. To block non-specific staining, slides were incubated with $10 \%$ normal donkey serum solution for $1 \mathrm{~h}$. Blocked sections were incubated in primary antibody overnight at $4^{\circ} \mathrm{C}$. Slides were then treated using an $\mathrm{LSAB}^{+}$kit (Dako Cytomation, Carpinteria, CA, USA), according to the manufacturer's recommended protocol. Slides were developed by incubating them with 3-amino-9ethyl carbazole and then counterstained with haematoxylin. A negative control was made by applying secondary antibody without primary antibody. Samples were evaluated by two pathologists who were blinded to patients' clinical information. Intensities were scored as 0 , no staining; 1 , weak; 2 , moderate; or 3 , strong.

Western blotting. Claudin 7 expression was also evaluated using Western blotting. Two normal gastric epithelium samples, 2 intestinal metaplasias, 2 gastric adenomas and 4 gastric carcinomas were used. Human gastric tissues were lysed [70 mM ß-glycerophosphate ( $\mathrm{pH} 7.2), 0.6 \mathrm{mM} \mathrm{Na}$ vanadate, $2 \mathrm{mM} \mathrm{MgCl}_{2}, 1 \mathrm{mM}$ EGTA, $1 \mathrm{mM}$ DTT, $0.5 \%$ Triton X-100, 0.5\% NP-40, $0.2 \mathrm{mM} \mathrm{PMSF}$ and protease inhibitors]. Protein $(50 \mu 1)$ was separated using $18 \%$ SDSPAGE and transferred onto a PVDF membrane (Millipore Corp., Billerica, MA, USA) for $1 \mathrm{~h}$. Membranes were blocked with 5\% non-fat milk in Tris-buffered saline/0.05\% Tween-20 (TBS-T) for $1 \mathrm{~h}$ at room temperature and probed with claudin 7-specific antibody (1:1000 dilution in $0.5 \%$ non-fat milk in TBS-T) overnight at $4^{\circ} \mathrm{C}$. After washing with TBS-T, membranes were treated with horseradish peroxidase-conjugated secondary antibody for $1 \mathrm{~h}$ and washed several times. Proteins were detected using an enhanced chemiluminescence system (Pierce, Rockford, IL, USA).

Statistical analysis. Claudin 7 in intestinal metaplasias, adenomas and cancers were compared to normal gastric epithelium using the Mann-Whitney U test. Claudin 7 expression was correlated to tumor cell differentiation, Lauren's classification, and tumor stage using Spearman methods. In all cases, $\mathrm{p}<0.05$ was considered statistically significant.

\section{Results}

Microarray data. Based on microarray data, genes with $>3$-fold change in expression levels $(\mathrm{p}<0.01)$ were selected: 2486 genes in diffuse-type gastric cancer tissue, 1200 genes in intestinal-type gastric cancer tissue, and 816 genes in mixed type gastric cancer tissue were up- or down-regulated. Among the genes with significant changes, claudin 4 and 7, epiplakin 1, 


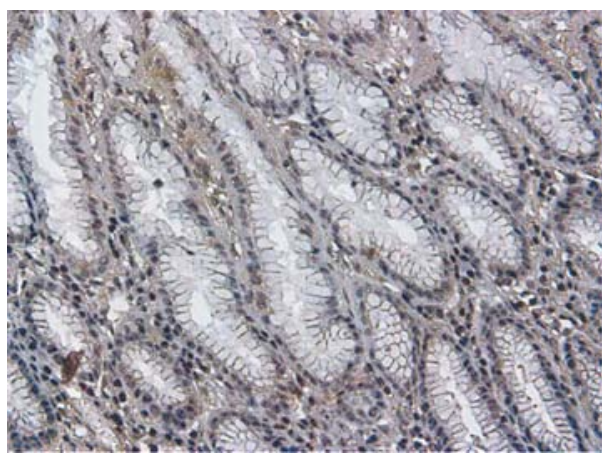

A

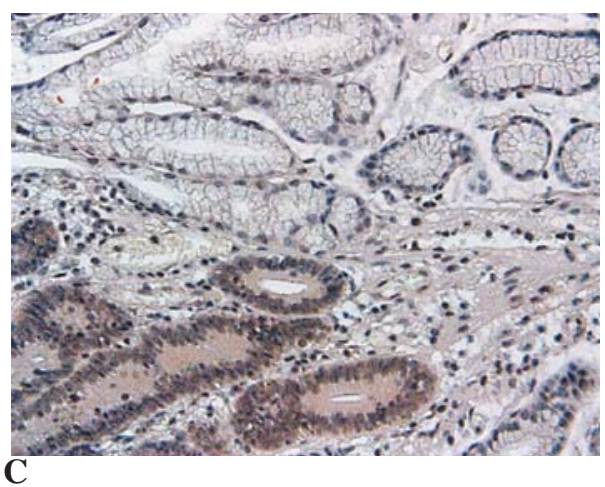

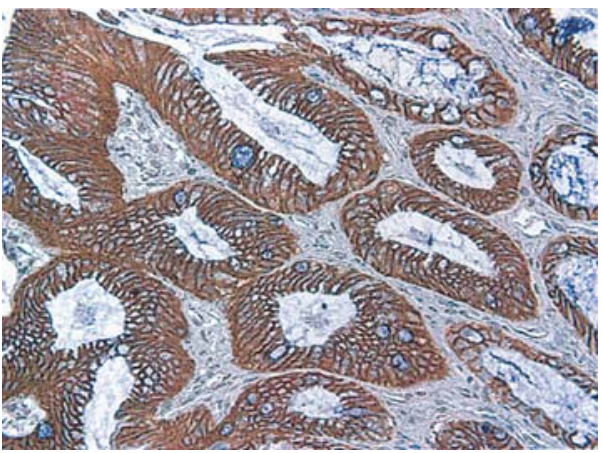

B

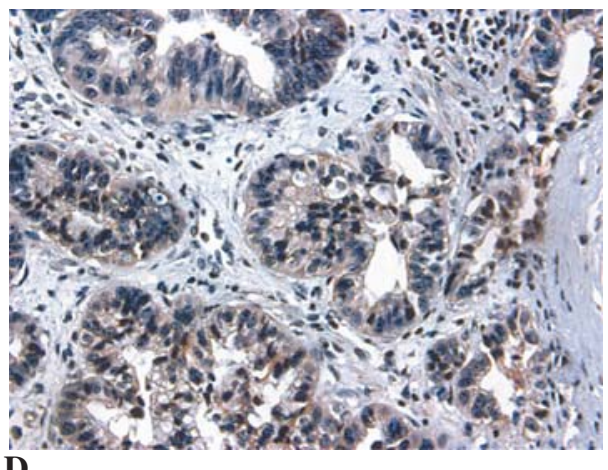

Figure 1. Representative immunohistochemical staining for claudin 7 in gastric epithelium, intestinal metaplasia, gastric adenoma and gastric carcinoma. (A) Claudin 7 expression in normal gastric epithelium; (B) intestinal metaplasia; (C) gastric adenoma; and (D) gastric carcinoma.

and anillin were up-regulated in intestinal-type gastric cancer. TTK protein kinase, thrombospondin 2, osteopontin, calgranulin $\mathrm{A}$ and $\mathrm{B}$, and cathepsin $\mathrm{B}$ and $\mathrm{C}$ were upregulated in diffuse-type gastric cancer. Compared to normal gastric epithelium, claudin 7 expression was 23.5-fold higher in gastric adenoma and 5.9-fold higher in intestinal-type gastric cancer. Claudin 7 expression was also slightly increased in diffuse- and mixed-type gastric cancer, but this change was not statistically significant.

Claudin 7 expression in gastric epithelium, intestinal metaplasia, adenoma and cancer. After immunohistochemical staining, most of the normal gastric epithelium did not express claudin 7, but some expressed it weakly at the basolateral membrane (Fig. 1A). The foveloar gland expressed claudin 7 . Claudin 7 was expressed homogeneously at cell-cell contacts and in the cytoplasm of intestinal metaplasias, adenomas and cancer cells. The basolateral membrane of intestinal metaplasia cells stained strongly (Fig. 1B). Claudin 7 was more often expressed in intestinal metaplasia than in gastric epithelium. Thirty $(52.6 \%)$ of 57 gastric epithelium samples and $50(86.2 \%)$ of 58 intestinal metaplasia samples expressed claudin 7 ( $<<0.001)$. Western blots showed that claudin 7 was expressed in intestinal metaplasia, but not in gastric epithelium (Fig. 2). Claudin 7 expression at the basolateral membrane of adenomas was not as prominent as in intestinal metaplasias (Fig. 1C). Claudin 7 was more often expressed in adenoma tissues than in gastric epithelium $(\mathrm{p}<0.05)$. Eleven $(91.7 \%)$ of 12 adenomas expressed claudin 7 . Cancer tissue also expressed claudin 7 more frequently than gastric epithelium $(\mathrm{p}<0.001)$. The expression pattern was similar to that in adenomas

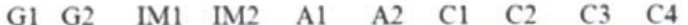

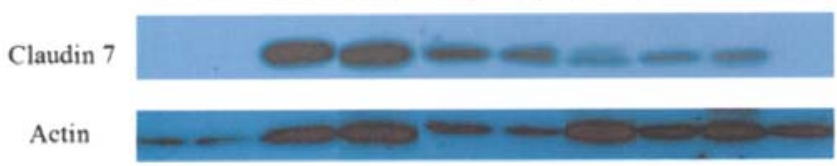

G1, 2: gastric epithelium; IM1, 2: intestinal metaplasia; A1, 2: adenoma; C1, 2, 3, 4: gastric cancer

Figure 2. Western blot of claudin 7 in normal gastric epithelium, intestinal metaplasia, gastric adenoma and gastric carcinoma. Claudin 7 was not expressed in normal gastric epithelium. In intestinal metaplasia, adenoma and carcinoma, it was well-expressed.

(Fig. 1D). Twenty-seven (47.4\%) of 57 gastric epithelium samples did not express claudin 7, while 129 (82.7\%) of 156 cancer samples did. However, there was no difference in the intensity or frequency of claudin 7 expression in cancer samples compared to intestinal metaplasias and adenomas ( $p>0.05$ and $>0.05$, respectively). Claudin 7 expression in gastric epithelium, intestinal metaplasia, adenoma, and cancer is summarized in Table I.

Association between claudin 7 expression, cancer cell differentiation and Lauren's classification. Poorly differentiated cancers associated with loss of claudin 7 ( $\mathrm{p}<0.05)$. Of 62 welldifferentiated cases, 5 (8.1\%) showed no claudin 7 expression, whereas $22(23.4 \%)$ of 94 poorly differentiated cases showed no expression (Table II).

Loss of claudin 7 expression occurred more often in diffuse-type gastric cancer than in intestinal-type $(\mathrm{p}<0.001)$. 
Table I. Claudin 7 expression in gastric epithelium, intestinal metaplasia, gastric adenoma and gastric carcinoma.

\begin{tabular}{|c|c|c|c|c|c|}
\hline & \multicolumn{4}{|c|}{ Cluadin 7 staining intensity } & \multirow[b]{2}{*}{ P-value } \\
\hline & None $(\%)$ & Weak $(\%)$ & Moderate (\%) & Strong (\%) & \\
\hline $\begin{array}{l}\text { Gastric epithelium } \\
(\mathrm{n}=57)\end{array}$ & $27(47.4)$ & $25(43.9)$ & $5 \quad(8.8)$ & $0 \quad(0)$ & \\
\hline $\begin{array}{l}\text { Intestinal metaplasia } \\
(\mathrm{n}=58)\end{array}$ & $8(13.8)$ & $31(53.4)$ & $17(29.3)$ & $2(3.4)$ & $<0.001$ \\
\hline $\begin{array}{l}\text { Adenoma } \\
(\mathrm{n}=12)\end{array}$ & $1 \quad(8.3)$ & $11(91.7)$ & $0 \quad(0)$ & $0 \quad(0)$ & $<0.05$ \\
\hline $\begin{array}{l}\text { Intestinal type cancer } \\
(\mathrm{n}=116)\end{array}$ & $13(11.2)$ & $66(56.9)$ & $33(28.4)$ & $4(3.4)$ & $<0.001$ \\
\hline $\begin{array}{l}\text { Diffuse type cancer } \\
(\mathrm{n}=34)\end{array}$ & $14(41.2)$ & $19(55.9)$ & $1 \quad(2.9)$ & $(0)$ & $>0.05$ \\
\hline
\end{tabular}

Table II. Association between cancer cell differentiation and claudin 7 expression.

\begin{tabular}{|c|c|c|c|c|c|}
\hline & \multicolumn{4}{|c|}{ Cluadin 7 staining intensity } & \multirow[b]{2}{*}{ P-value } \\
\hline & None $(\%)$ & Weak (\%) & Moderate (\%) & Strong (\%) & \\
\hline $\begin{array}{l}\text { Well-differentiated } \\
(\mathrm{n}=62)\end{array}$ & $5 \quad(8.1)$ & 37 (59.7) & $17(27.4)$ & $3(4.8)$ & \\
\hline $\begin{array}{l}\text { Poorly differentiated } \\
(\mathrm{n}=94)\end{array}$ & $22(23.4)$ & $51(54.3)$ & 19 (20.2) & $2(2.1)$ & $<0.05$ \\
\hline
\end{tabular}

Only $13(11.2 \%)$ of 116 intestinal-type samples lacked claudin 7 , but $14(41.2 \%)$ of 34 diffuse-type samples showed no expression. In 37 (31.8\%) of 116 intestinal-type cancer cases, claudin 7 immunoreactivity was moderate or strong, but only $1(2.9 \%)$ of 34 cases with diffuse-type cancer showed moderate intensity. Compared to normal gastric epithelium, intestinal-type gastric cancer more often expressed claudin 7 , but diffuse-type did not $(\mathrm{p}<0.001$ and $>0.05$, respectively; Table I).

Association between claudin 7 expression and tumor stage. Claudin 7 expression did not change with tumor progression (Table III). The percentage of cases that lacked claudin 7 expression in each cancer stage was consistent. In intestinaltype cancer, $6(12.5 \%)$ of 49 stage I cases and $2(13.3 \%)$ of 15 stage IV cases showed no claudin 7 expression ( $>0.05)$. In diffuse-type cancer, $6(50.0 \%)$ of 12 stage I cases and 3 $(33.3 \%)$ of 9 stage IV cases lacked claudin 7 expression $(\mathrm{p}>0.05)$.

\section{Discussion}

Tight junction proteins are suspected to play an important role in cancer progression, invasion, and metastasis, but there are few studies reporting on the role of tight junction proteins in these processes. Gastric carcinoma is the fourth most frequently diagnosed cancer (19), but studies of the tight junction in gastric carcinoma are rare. In this study we were able to show the expression pattern of claudin 7 in each carcinogenic step of gastric carcinoma. In addition, we demonstrated its clinical implications.

Claudin 7 up-regulation is an early event in gastric tumorigenesis, based on results showing claudin 7 overexpression in gastric dysplasia and cancer (16). According to our results, significant claudin 7 expression was already noted in intestinal metaplasias, suggesting that up-regulation may have occurred earlier. This result was slightly different from previous studies showing weak claudin 7 expression in intestinal metaplasia (16). This difference may have been caused by the number of cases studied. Our study included 58 cases of intestinal metaplasia, and the former study had 3 cases.

Our result agreed with a previous study demonstrating that diffuse-type gastric adenocarcinoma did not express as much claudin 7 as intestinal-type tumors (16). Also, compared to normal gastric epithelium, intestinal-type gastric cancer expressed claudin 7 more often, but diffuse-type did not. These results may account for different carcinogenic processes and behaviors of intestinal and diffuse-type gastric carcinomas. 
Table III. Association between claudin 7 expression and tumor stage.

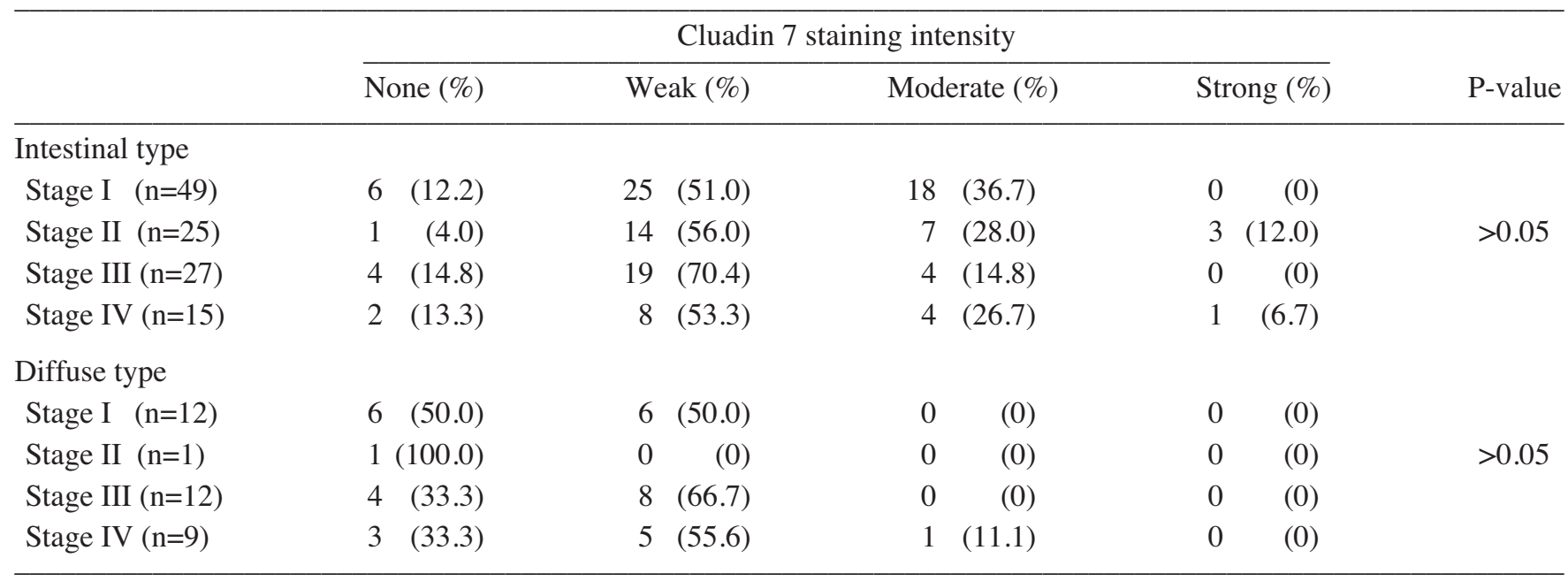

They also support the carcinogenesis process from intestinal metaplasia to adenoma to intestinal-type gastric cancer because all three expressed claudin 7. It explains why diffusetype gastric cancer does not express claudin 7 , since it develops from gastric epithelium, not intestinal metaplasia (20).

In esophageal and breast cancer, similar results to ours were reported $(6,21)$. Poorly differentiated cancer was associated with loss of claudin 7. However, loss of claudin 7 expression correlated with tumor progression. In gastric cancer, claudin 7 expression was maintained regardless of tumor stage, especially for intestinal-type gastric cancer where only $10 \%$ of each tumor stage did not express claudin 7. Claudin 7 up-regualtion might be an early event in carcinogenesis, but it was not lost in advanced stages.

Like claudin 7, claudins 1, 3, 4 and 5 were also more frequently expressed in intestinal-type gastric cancer, and their expression was not associated with tumor stage $(5,22)$. In addition, tumors expressed claudin 7 more often than the surrounding normal gastric epithelium. Claudin 1 expression was strong in gastric cancer, but it was also expressed in non-neoplastic gastric mucosa. Claudins 3 and 4 were more selectively expressed in gastric cancer (5). These results suggest that claudins 3, 4 and 7 may be potential therapeutic targets for gastric cancer. Monoclonal antibody against these claudins, complexed with anti-cancer agents may be able to attack gastric cancer cells selectively $(4,5)$. More research is needed to validate such treatment.

Understanding tissue- and cancer-specific expression of tight junction proteins could lead to new cancer diagnostic and therapeutic targets. In this study we showed that claudin 7 expression changed during gastric carcinogenesis. Claudins may be potential targets for antibody-based cancer treatment. More studies of the expression patterns and function of tight junction proteins and their underlying role in gastric cancer are warranted.

\section{Acknowledgements}

This research was supported by the Yonsei University College of Medicine, Internal Medicine Research Grant 6-2005-0011 and the Intelligent Microsystem Center (IMC; http://www. microsystem.re.kr) F0001004-2006-31, which carries out one of the 21st century's Frontier R\&D Projects sponsored by the Korea Ministry of Commerce, Industry and Energy.

\section{References}

1. Aijaz S, Balda MS and Matter K: Tight junctions: molecular architecture and function. Int Rev Cytol 248: 261-298, 2006.

2. Rahner C, Mitic LL and Anderson JM: Heterogeneity in expression and subcellular localization of claudins 2,3,4 and 5 in the rat liver, pancreas and gut. Gastroenterology 120: 411-422, 2001.

3. Chlenski A, Ketels KV, Korovaitseva GI, Talamonti MS, Oyasu R and Scarpelli DG: Organization and expression of the human zo-2 gene (tjp-2) in normal and neoplastic tissues. Biochim Biophys Acta 1493: 319-324, 2000.

4. Morin PJ: Claudin proteins in human cancer: promising new targets for diagnosis and therapy. Cancer Res 65: 9603-9606, 2005.

5. Resnick MB, Gavilanez M, Newton E, et al: Claudin expression in gastric adenocarcinomas: a tissue microarray study with prognostic correlation. Hum Pathol 36: 886-892, 2005.

6. Kominsky SL, Argani P, Korz D, et al: Loss of the tight junction protein claudin 7 correlates with histological grade in both ductal carcinoma in situ and invasive ductal carcinoma of the breast. Oncogene 22: 2021-2033, 2003.

7. Al Moustafa AE, Alaoui-Jamali MA, Batist G, et al: Identification of genes associated with head and neck carcinogenesis by cDNA microarray comparison between matched primary normal epithelial and squamous carcinoma cells. Oncogene 21: 2634-2640, 2002.

8. Hough CD, Sherman-Baust CA, Pizer ES, et al: Large-scale serial analysis of gene expression reveals genes differentially expressed in ovarian cancer. Cancer Res 60: 6281-6287, 2000.

9. Long H, Crean CD, Lee WH, Cummings OW and Gabig TG: Expression of Clostridium perfringens enterotoxin receptors claudin-3 and claudin-4 in prostate cancer epithelium. Cancer Res 61: 7878-7881, 2001

10. Michl P, Buchholz M, Rolke M, et al: Claudin-4: a new target for pancreatic cancer treatment using Clostridium perfringens enterotoxin. Gastroenterology 121: 678-684, 2001.

11. Cheung ST, Leung KL, Ip YC, et al: Claudin-10 expression level is associated with recurrence of primary hepatocellular carcinoma. Clin Cancer Res 11: 551-556, 2005

12. Michl P, Barth C, Buchholz M, et al: Claudin-4 expression decreases invasiveness and metastatic potential of pancreatic cancer. Cancer Res 63: 6265-6271, 2003.

13. Sato N, Fukushima N, Maitra A, et al: Gene expression profiling identifies genes associated with invasive intraductal papillary mucinous neoplasms of the pancreas. Am J Pathol 164: 903-914, 2004. 
14. Fujita H, Chiba H, Yokozaki H, et al: Differential expression and subcellular localization of claudin $7,-8,-12,-13$ and -15 along the mouse intestine. J Histochem Cytochem 54: 933-944, 2006.

15. Sauer T, Pedersen MK, Ebeltoft K and Naess O: Reduced expression of Claudin 7 in fine needle aspirates from breast carcinomas correlate with grading and metastatic disease. Cytopathology 16: 193-198, 2005.

16. Johnson AH, Frierson HF, Zaika A, et al: Expression of tight junction protein claudin 7 is an early event in gastric tumorigenesis. Am J Pathol 167: 577-584, 2005.

17. Schena M, Shalon D, Davis RW and Brown PO: Quantitative monitoring of gene expression patterns with complementary DNA microarray. Science 270: 467-470, 1995.
18. Eisen MB, Spellman PT, Brown PO and Boststein D: Cluster analysis and display of genome-wide expression patterns. Proc Natl Acad Sci USA 95: 14863-14868, 1998.

19. Parkin DM, Bray F, Ferlay J and Pisani P: Estimating the world cancer burden: Globocan 2000. Int J Cancer 94: 153-156, 2001.

20. Yuasa Y: Control of gut differentiation and intestinal-type gastric carcinogenesis. Nat Rev Cancer 3: 592-600, 2003.

21. Usami Y, Chiba H, Nakayama F, et al: Reduced expression of claudin-7 correlates with invasion and metastasis in squamous cell carcinoma of the esophagus. Hum Pathol 37: 569-577, 2006.

22. Soini Y, Tommola S, Helin H and Martikainen P: Claudins 1, 3, 4 and 5 in gastric carcinoma, loss of claudin expression associates with the diffuse subtype. Virchows Arch 448: 52-58, 2006. 